\title{
The salt secretion of leaves promotes the competitiveness of Reaumuria soongarica in a desert grassland
}

Chang-shun Wang ${ }^{1,2+}$, Hui-qing Wang ${ }^{1,3+}{ }^{+}$Wei Wang ${ }^{1}$, Cun-zhu Liang ${ }^{1}$, Hua-min Liu ${ }^{1}$ and Li-xin Wang ${ }^{1 *}$

\begin{abstract}
Background: For better understanding the mechanism of Reaumuria soongarica community formation in a salt stressed grassland ecosystem, we designed a field experiment to test how leaves salt secretion changes the competitive relationship between species in this plant communities.

Results: Among the three species (R. soongarica, Stipa glareosa and Allium polyrhizum) of the salt stressed grassland ecosystem, the conductivity of $R$. soongarica rhizosphere soil was the highest in five soil layers (0-55 cm depth). The high soil conductivity can increase the daily salt secretion rate of plant leaves of $R$. soongarica. In addition, we found the canopy size of $R$. soongarica was positively related to the distance from S. glareosa or A. polyrhizum. The salttolerance of $R$. soongarica was significantly higher than the other two herbs (S. glareosa and A. polyrhizum). Moreover, there was a threshold $(600 \mu \mathrm{S} / \mathrm{cm})$ for interspecific competition of plants mediated by soil conductivity. When the soil conductivity was lower than $600 \mu \mathrm{S} / \mathrm{cm}$, the relative biomass of $R$. soongarica increased with the soil conductivity increase.
\end{abstract}

Conclusions: The efficient salt secretion ability of leaves increases soil conductivity under the canopy. This leads the formation of a "saline island" of R. soongarica. Meanwhile R. soongarica have stronger salt tolerance than S. glareosa and A. polyrhizum. These promote the competitiveness of $R$. soongarica and inhibit interspecies competition advantage of the other two herbs (S. glareosa and A. polyrhizum) in the plant community. It is beneficial for R. soongarica to establish dominant communities in saline regions of desert grassland.

Keywords: Stress tolerance, Interspecies competition, Tradeoff, Soil conductivity, Inner Mongolia

\section{Background}

There are two sides of the same coin between species establishment and community stability [1-3]. The zonal plant communities alternate along environmental gradients [4] and have clear and stable boundaries $[5,6]$. However, the typical highly drought-tolerant desert plant Reaumuria soongarica has a unique distribution [7]. $R$.

\footnotetext{
*Correspondence: Ix_wimu@163.com

${ }^{\dagger}$ Chang-shun Wang and Hui-qing Wang contributed equally to this work.

${ }^{1}$ School of Ecology and Environment, Inner Mongolia University,

Hohhot 010021, Inner Mongolia, China

Full list of author information is available at the end of the article
}

soongarica not only dominates desert communities in Asia, but also forms communities in the eastern Mongolian Plateau grassland where soils are generally saline [8]. Therefore, $R$. soongarica has managed not only adapt to the extreme drought of the desert environment but also occupy areas with high moisture, where other plants dominate with different strategies across vegetation zones [7]. Generally, a zonally dominant species cannot spread to other vegetation zones [9]. This raises the question that how $R$. soongarica breaks this general rule?

In reality, some plant species cannot adapt to environmental stress, whereas others that have adapt to a stressed environment cannot spread to areas with greater 
precipitation or higher temperature regimes [10]. For example, the desert steppe prevents the establishment of desert plant seedlings via strong interspecific competition imposed by its dominant populations, which maintains the relative stability of the vegetation distribution $[11,12]$. Conversely, steppe plants have difficulty adapting to harsh desert habitats and cannot effectively survive in the desert $[13,14]$. This phenomenon is the result of the combined effects of plant physiological tolerance to abiotic stress and interspecies competition (i.e., the ability of a plant to inhibit the growth of another plant) [15]. It remains unclear how the environmental tolerance and interspecies competition of plants act on establishment of $R$. soongarica in saline regions within the steppe.

$R$. soongarica is able to secreted $\mathrm{Na}^{+}$through salt gland to alleviate the toxic effects of $\mathrm{Na}^{+}$and maintain water status in plant, which contribute to stimulate the growth of plant [16]. The secrete salt from the leaves of $R$. soongarica deposit on the ground [17]. This is similar to Mesembryanthemum crystallinum that can absorb salt from deeper soil layers and deposit the salt on the soil surface, and lead to the death of neighboring, less salttolerant plants, and ultimately form a dominant community [18]. Owing to the limited tolerance of a given plant to its environment, this interspecies competition advantage is not identical across environments $[19,20]$.

The tolerance of plants to extreme environments are based on specialized functions [21, 22]. In a stress-less environment, these special functions are redundant and may become a burden on interspecific competition [23]. Thus, plants with higher tolerance will show a reduced ability in terms of access to resources under interspecies competition in a stress-free environment $[3,24]$. We assumed that $R$. soongarica was unable to occupy areas with better environmental conditions (i.e., vast grassland areas, not just salty areas) because it cannot compete with local dominant species, especially herbs.

Our study aimed to answer this question by focusing on the adaptability of $R$. soongarica to stress and interspecies competition in a grassland community. Therefore, the first hypothesis of the present study is that whether the soil salinity under $R$. soongarica is higher than that of herbs, and whether the presence of $R$. soongarica affects the position of the herb. The second hypothesis of the present study is that $R$. soongarica is unable to compete with dominant plants in grassland areas under conditions of low soil salinity.

\section{Results}

Soil conductivity and the plant community distribution The soil conductivity (soil salinity) increases with the decrease of elevation, which produces clear boundaries between the plant communities in the basin (Fig. 1). Outside the ancient lake basin is the zonal plant community, which is dominated by Stipa glareosa. From the basin margin to the center, the plant communities are $R$. soongarica $+S$. glareosa, $R$. soongarica + Allium polyrhizum, pure $R$. soongarica, $R$. soongarica + Kalidium foliatum, and pure $K$. foliatum.

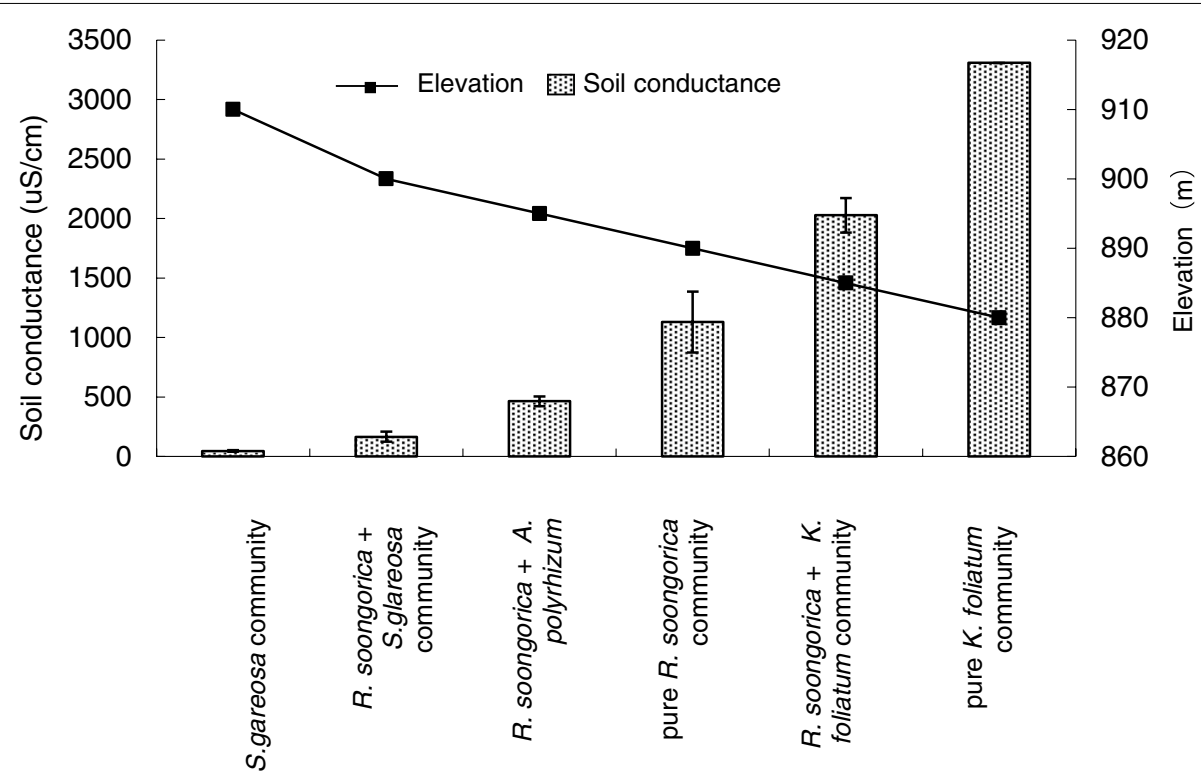

Fig. 1 Mean soil conductivity $(0-55 \mathrm{~cm})$ and elevation in different communities at the research site. The vertical lines at the top of the bars represent the standard deviation $(\mathrm{N}=3)$ 
Rhizosphere soil conductivity of the different plant species The rhizosphere soil conductivity of $R$. soongarica, $S$. glareosa, and $A$. polyrhizum increased with the increase of soil depth (Fig. 2). R. soongarica had significantly higher rhizosphere soil conductivity than $S$. glareosa and $A$. polyrhizum in all soil depths $(P \leq 0.001)$ (Fig. 2$)$. In $R$. soongarica and $S$. glareosa community, S. glareosa showed the lowest rhizosphere soil conductivity (Fig. 2A).

\section{Salt secretion rate of $R$. soongarica}

There were significant differences in leaf salt secretion rates among the three communities $(P<0.01)$. The salt secretion rate increased with the increase of soil conductivity (Fig. 3). The daily salt secretion rate ranged from 1 to $2 \%$ of the fresh leaf weight in different communities. The amount of salt excreted increased over time.

\section{Population distribution patterns in the community}

With an increase of the canopy size of $R$. soongarica, $S$. glareosa and A. polyrhizum were situated further away from the shrub (Fig. 4). The correlation between the $R$. soongarica canopy diameter and the distance to the nearest $S$. glareosa $\left(R^{2}=0.4065 ; P<0.05\right)$ was higher than that to the nearest $A$. polyrhizum $\left(R^{2}=0.1256 ; P<0.05\right)$.

\section{Effect of salinity on plant growth}

Soil conductivity had a negative effect on plant growth (Fig. 5A). The plant relative biomass decreased with an increase of the soil conductivity, and the relative biomass of $R$. soongarica decreased more slowly than S. glareosa and $A$. polyrhizum $(P<0.001, N=54)$. The relative biomass of the two herbs at $600 \mu \mathrm{S} / \mathrm{cm}$ was decreased to less than $50 \%$, whereas that of $R$. soongarica was decreased to $80 \%$. The relative biomass of the shrub was decreased to $50 \%$ at $2000 \mu \mathrm{S} / \mathrm{cm}$, whereas that of the two herbs fell almost to zero under the same conditions. At low soil conductivity ( 200 or $600 \mu \mathrm{S} / \mathrm{cm}$ ), the relative biomass of $S$. glareosa decreased slightly faster than that of $A$. polyrhizum; therefore, $A$. polyrhizum was more salt-tolerant than S. glareosa.

\section{Effect of interspecies competition on the growth of $R$. soongarica}

The relative biomass of $R$. soongarica decreased significantly when planted with S. glareosa or A. polyrhizum at low soil salinity (soil conductivity below $600 \mu \mathrm{S} / \mathrm{cm}$; $P<0.001$; Fig. 5B). However, there was no significant difference in relative biomass between single planting $R$. soongarica and mixed planting at high soil salinity (soil conductivity over $1000 \mu \mathrm{S} / \mathrm{cm} ; P=0.1$ ). The herbs significantly limited the growth of $R$. soongarica seedlings at low soil conductivity $(\leq 600 \mu \mathrm{S} / \mathrm{cm})$; however, their effect was eliminated at higher soil conductivity $(\geq 1000 \mu \mathrm{S} /$ $\mathrm{cm})$, which was detrimental to the herbs.

\section{Discussion}

\section{Competitiveness and environment}

Processes related to special functions can alter the local environment. Thus, the limited competitiveness of the

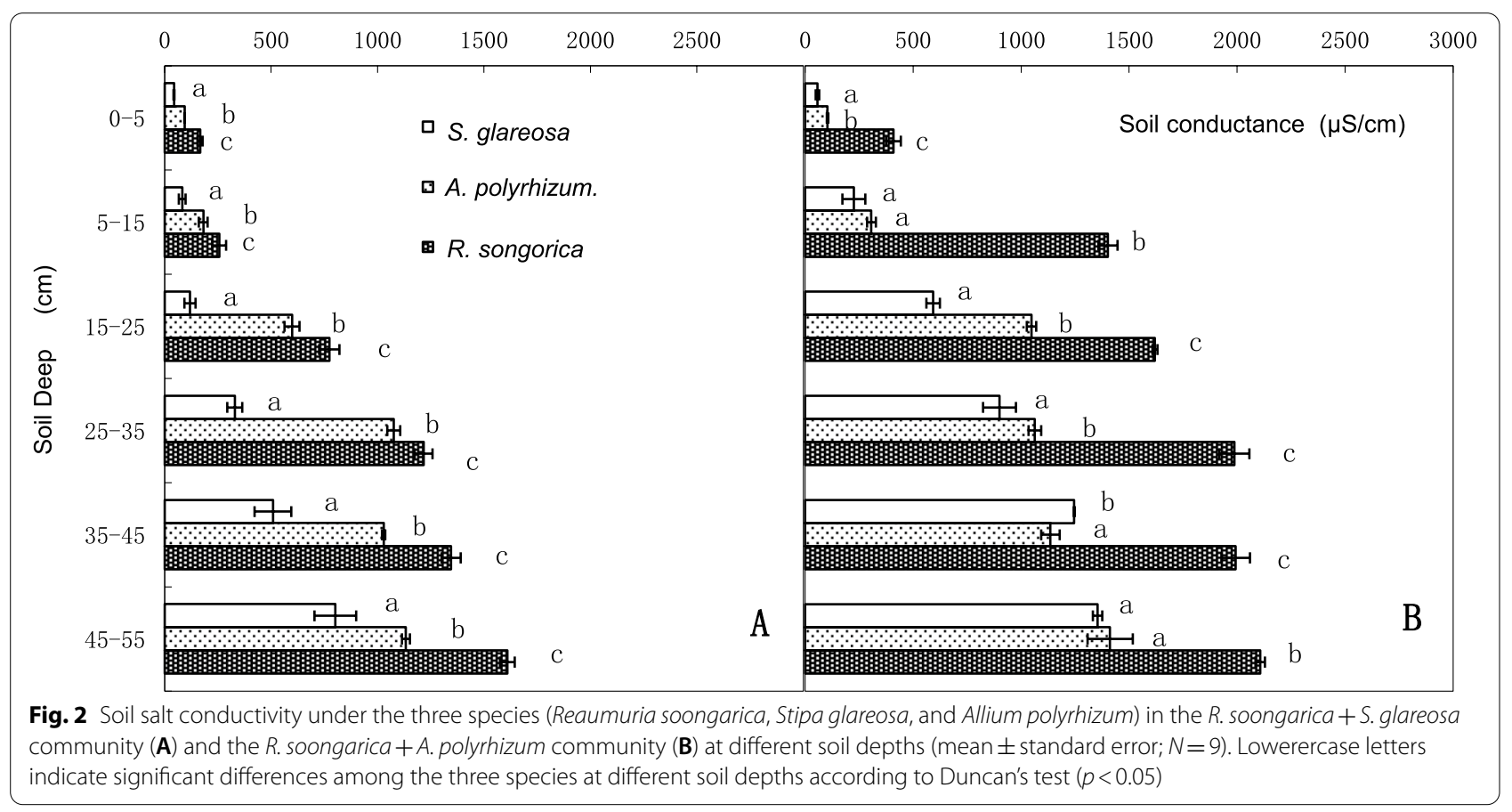




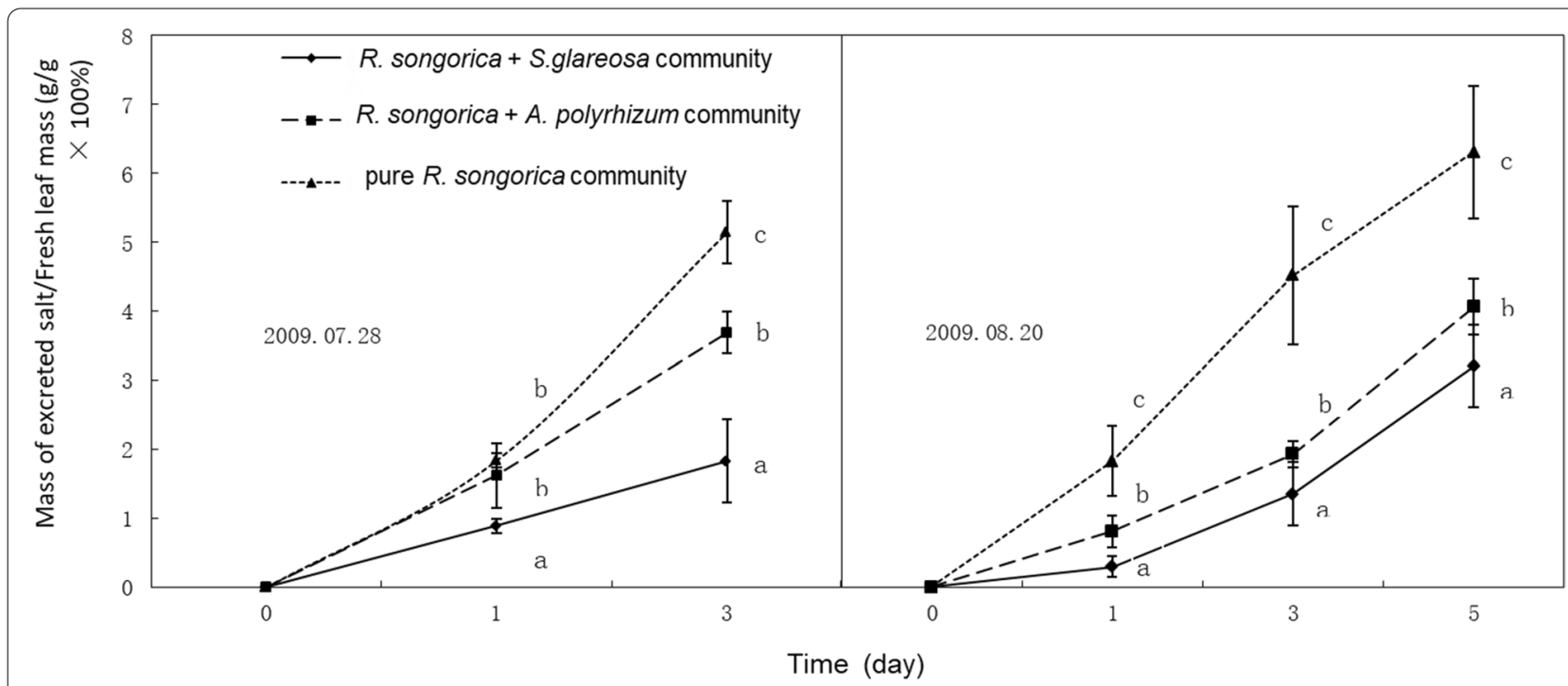

Fig. 3 Quantitative analysis of salt excreted by R. soongarica in different communities (R. soongarica +S. glareosa, R. soongarica +A. polyrhizum, and pure R. soongarica) with different soil conductivities at two different times (mean \pm standard error; $N=9$ ). Lowercase letters indicate significant differences among the communities on a different day according to Duncan's test $(p<0.05)$

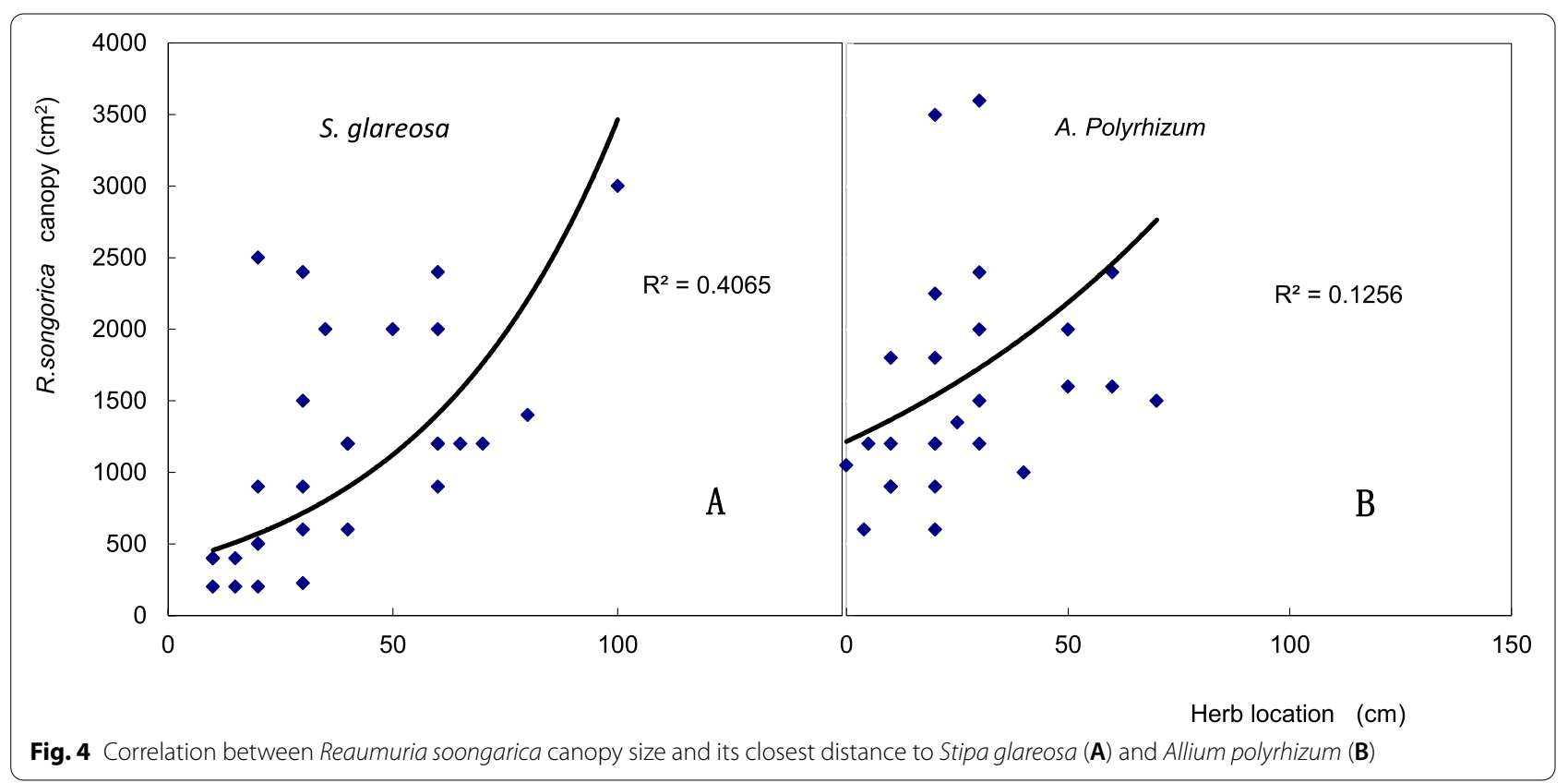

original vegetation [25-27] is important for the successful establishment of plant species in the community $[2,3]$. Our results showed that the conductivity of every soil layer was significantly higher for $R$. soongarica than for the two herbs (Fig. 2) and the higher the soil conductivity, the higher the salt secretion of $R$. soongarica (Fig. 1 and 3). Therefore, the physiological process of salt secretion from $R$. soongarica leaves created the spatial heterogeneity of soil salt in the coexisting community, which was important for its establishment in the grassland.

A plant can inhibit the growth of its competitors by developing special physiological functions, thus to enhance its ability to obtain resources and improve species competitiveness $[28,29]$. In our study, $R$. soongarica rhizosphere soil had high electrical conductivity, forming a "saline island". This may be due to the strong salt secretion ability of $R$. soongarica leaves (Fig. 3). 


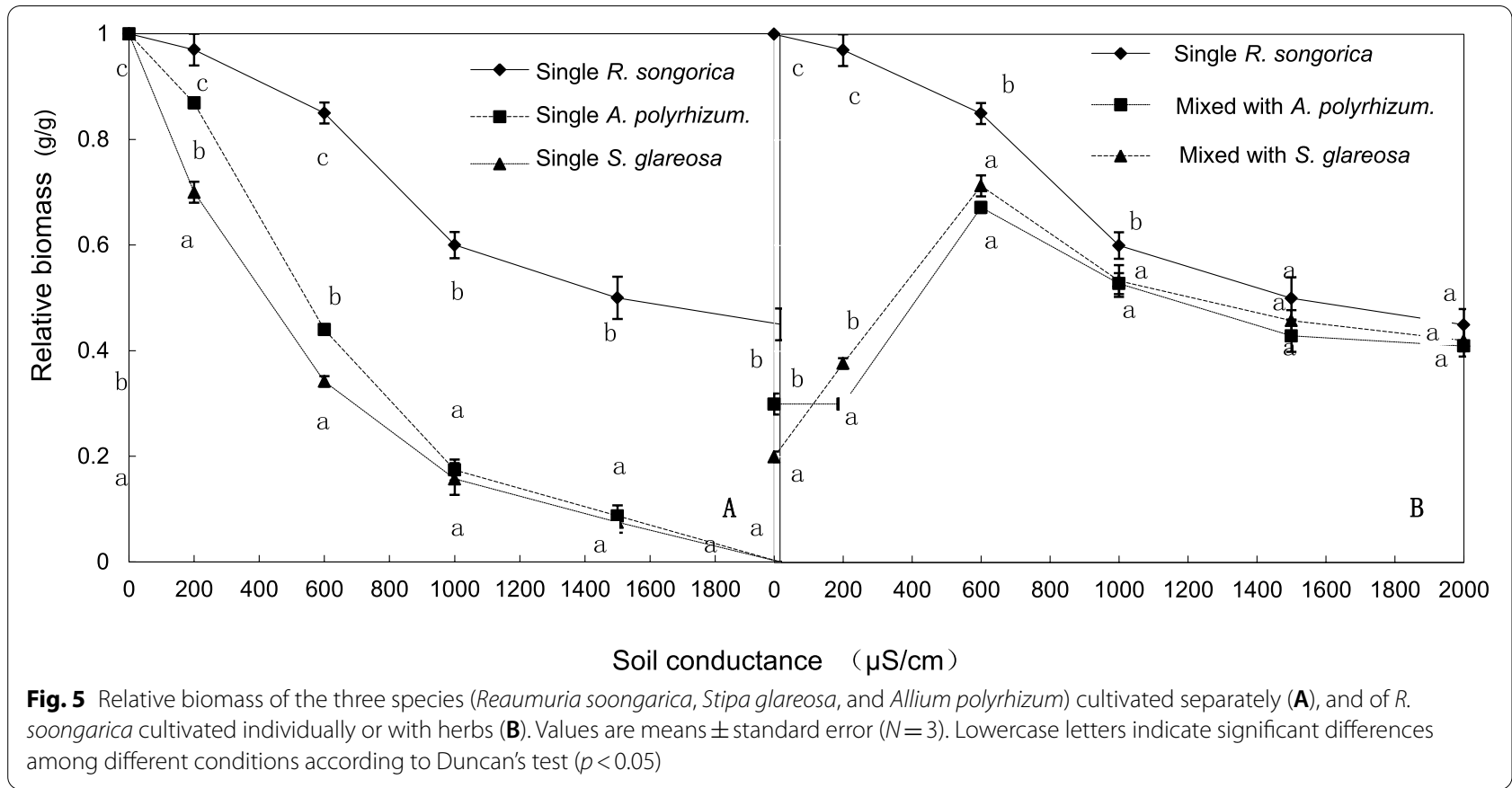

Moreover, the increase of $R$. soongarica canopy can promote the growth of saline island, and drive away plant species with low salt tolerance. This may be the reason why $S$. glareosa and A. polyrhizum are planted far away from $R$. soongarica (Fig. 4). Our results support first the hypothesis that salt secreted by $R$. soongarica leaves can change the soil salinity and inhibit the growth of plant species with low salt tolerance, thereby to change the structure of plant community.

Plants can gain higher competitiveness through allelopathy [30], which increases their chances of establishment [23]. This phenomenon is not always the result of a specialized physiological behavior. For example, oak leaves can inhibit the growth of herbaceous plants [31] and the removal of alpine forest litter can considerably promote the growth of herbs [32]. Thus, the ability to change the environment (i.e., allelopathy) is key point for the survival of plants in this community; it changes the level of interspecies competition and ultimately leads to changes in the community structure.

\section{Competitiveness and tolerance}

Many drought- or salt-tolerant species grow better in a stress-free environment $[25,33]$; however, the realized niche of most stress-tolerant plants often deviates from their fundamental niche because of interspecies competition [15]. The results of our pot experiment showed that the relative biomass of $R$. soongarica, S. glareosa and $A$. polyrhizum decreased in the soil environment with high conductivity. In other words, these three plant species did not depend on salt physiologically, and preferred the environment without salt stress (Fig. 5A). Additionally, the effect of soil salinity on the growth of the two herbs was much greater than that of $R$. soongarica. That is, $R$. soongarica had better tolerance to high salinity environment.

In our pot experiment, the competitiveness of $R$. soongarica is not strong enough, and the growth of its seedlings is inhibited by S. glareosa and A. polyrhizum in the low salinity soil environment (Fig. 5B). On the desert steppe in eastern Inner Mongolia, the soil salinity is not extremely high. The shrub species ( $R$. soongarica) with more salt tolerance had no interspecific competitive advantage with herbs (S. glareosa and A. polyrhizum), and could not establish a wide range of dominant communities [7]. Although $R$. soongarica can adapt to drought and salinity, it has no interspecific competitive advantage in low salinity soil environment. A species with both high stress resistance and interspecies competitiveness will reduce species diversity in communities. However, this is not consistent with the result of long-term evolution. In fact, plants may tradeoff between different adaptive abilities (e.g., tolerance and interspecies competitiveness, or acquisitive and conservative) [34, 35]. Many studies have also confirmed the exclusive relationship between environmental tolerance and resources competitiveness [25]. At low soil nutrient levels, species with high tolerance are more successful. Conversely, when the soil nutrient level 
is high, the same species are at a competitive disadvantage [36]. Environmental pressure limits the competitiveness of species with strong resource acquisition ability, which in turn increases the competitiveness of highly stress resistant species [24]. R. soongarica has evolved salt glands to adapt to drought in desert regions or highsalinity grasslands. However, this specialized trait and capability provides no advantage in non-specific environment. And the plant becomes powerless when competing with dominant grassland species without these specializations [37].

Successful species establishment is easy to understand based on the assumption that the competitiveness of the species distributed across zones is greater than that of the native species $[3,27]$. However, the long-term presence of the vegetation zone proves that this assumption is not universally true [35]. Our results support another view that competitiveness is not "real," but a process related to special functions [28]. The ecological mechanisms of $R$. soongarica establishment, which rely on salt secretion, facilitate the spread of $R$. soongarica across vegetation zones and the formation of a community on the desert steppe in eastern Inner Mongolia. The soil conductivity of the saline islands (Fig. 2) was high enough to inhibit herb growth (Fig. 5A), and $R$. soongarica grown on a saline island could maintain its growth environment thereby to cope with the competition of herbs (Fig. 5B). Our results add new knowledge to the existing literature, and thus help to advance our understanding of how some shrub species with strong environmental tolerance can survive in desert steppes and establish a stable community. The interspecies competition advantage of a species is inverse to its ability to tolerate abiotic stress, indicating a tradeoff among different abilities, which determines the distribution of plants.

\section{Conclusion}

Our results showed that $R$. soongarica has strong interspecific competitive advantage in high salinity soil environment and can establish a stable dominant community. First, $R$. soongarica can tolerate strong environmental salt stress. Second, the salt secreted by $R$. soongarica leaves can affect soil conductivity and increase soil salinity to form a salt island. The competitive advantage of herb species (S. glareosa and A. polyrhizum) over $R$. soongarica will be reversed under the condition of high soil salinity. This work is helpful to understand how shrub species with strong environmental tolerance survive and establish stable dominant communities in grassland. Our findings support the hypothesis that the interspecies competition advantage of a species is inverse to its ability to tolerate abiotic stress, indicating a tradeoff among different abilities, which determines the distribution of plants.

\section{Methods}

\section{Experimental location}

Our study site was located in an ancient lake basin in the east of Erlianhot City, Inner Mongolia, China (43⒉ $23^{\prime} 16^{\prime \prime}$ $43^{\circ} 42^{\prime} 28^{\prime \prime} N, 112^{\circ} 01^{\prime} 1^{\prime \prime}-112^{\prime} 01^{\prime} 78^{\prime \prime}$ E; 910 m elevation) (Additional File 1, 2, 3, 4, 5 and 6 ).From 1971 to 2009, mean annual temperature was $4.4{ }^{\circ} \mathrm{C}$ with the lowest in January $\left(-17.7^{\circ} \mathrm{C}\right)$ and highest $\left(-23.7^{\circ} \mathrm{C}\right)$ in July, and mean annual precipitation was $137.0 \mathrm{~mm}$ (about $67.2 \%$ falling in June and August) The diameter of the basin is approximately $15 \mathrm{~km}$ and the maximum depth is $30 \mathrm{~m}$. The study area has a grazing history with light intensity, and the most common large herbivores are sheep and camels.

The local government authorized us to collect plant samples. The voucher specimen with the collect number (Zhao 20,080,705) was identified by Professor Liqing Zhao and deposited in Herbarium, Inner Mongolia College (HIMC).

\section{Experimental design Soil conductivity}

In order to test the difference of soil conductivity (soil salinity) in distinct herb and shrub coexisting communities, we collected rhizosphere soil samples from each of three plant species in $R$. soongarica and S. glareosa community and $R$. soongarica and A. polyrhizum community separately. The sampled soil layers were $0-5 \mathrm{~cm}$, $5-15 \mathrm{~cm}, 15-25 \mathrm{~cm}, 25-35 \mathrm{~cm}, 35-45 \mathrm{~cm}$, and $45-55 \mathrm{~cm}$. Each soil sample had three replicates. A total of 108 soil samples were collected. Soil conductivity was measured using the conductance method (Multi 340i; WTW Xylem Analytics, Weilheim, Germany).

\section{Salt secretion rate}

To detect the difference of leaves salt secretion rate of $R$. soongarica in the distinct communities, we selected 3 individual $R$. soongarica from three types of plant communities ( $R$. soongarica and $S$. glareosa, $R$. soongarica and $A$. polyrhizum, and pure $R$. soongarica community) separately, and each community type has three replicates. One branch per individual was marked randomly. At the beginning of the experiment, the leaf surface of the selected branches was washed repeatedly with distilled water to ensure that the surfaces were salt-free. The salt secretion rate was measured on the first, third, fifth, and seventh day. Each marked branch was soaked in $150 \mathrm{~mL}$ distilled water, and used a conductivity meter (Multi 340i; WTW Xylem Analytics) to measure the conductivity of solution. Subsequently, branches were harvested to measure the weight of the fresh leaves using an electronic balance (YP1002N; INESA, Shanghai, China) The experiment was conducted in July 2009 and repeated in August 
Because herbivore grazing damaged the marked plants, we only obtained two and three complete datasets in July and August, respectively.

\section{Population distribution patterns}

The amount of salt secretion was found to correlate positively with the crown size of $R$. soongarica. The salt secreted by the $R$. soongarica leaves was likely to increase the soil conductivity, and thus affect the growth of the herbs in the community. This could be demonstrated by the canopy area of $R$. soongarica and the distance between $R$. soongarica plants and the neighboring herbs. To investigate the effect of the salt secretion of the $R$. soongarica leaves on neighbor species, we selected $30 R$. soongarica randomly, measured their canopy diameter and the distances to nearest $S$. glareosa and A. polyrhizum in the $R$. soongarica and A. polyrhizum community The crown is assumed to be a perfect circle, and its area is calculated by the diameter.

\section{Laboratory cultivation experiment}

To verify the changes of competitiveness of different plants under different salt conditions, we conducted a cultivation experiment in laboratory. $R$. soongarica, S. glareosa, and A. polyrhizum seeds were collected in August 2008, and cultured in March of the next year. First, the seeds were soaked in distilled water for $30 \mathrm{~min}$ and treated with $0.1 \%$ gibberellin to break the seed dormancy. Then the seeds were placed between double filter papers and germinated in a Petri dish in a dark environment. Finally, the germinated seeds were transplantedinto a paper cup $(12 \mathrm{~cm}$ height, $7 \mathrm{~cm}$ top diameter, and $5 \mathrm{~cm}$ bottom diameter) filled with $300 \mathrm{~g}$ of river sand (rinsed repeatedly with distilled water to remove any salt and nutrient. Five combinations of three species were prepared: the first was pure $S$. glareosa with six seedlings arranged in a ring at $1 \mathrm{~cm}$ from the cup wall, the second was pure $A$. polyrhi$z u m$, the third was pure $R$. soongarica with only one seedling in the center of the cup. The other two were to place six $S$. glareosa or A. polyrhizum seedlings around one $R$. soongarica respectively (the ratio based on field observation).

Based on local precipitation and soil nutrient levels The seedlings were cultivated in an artificial climate chamber (ZRX-1000 ESW; TESTMART, Hangzhou, China) at $25{ }^{\circ} \mathrm{C}$ and $70 \%$ relative humidity with a $14 \mathrm{~h} / 10 \mathrm{~h}$ light/dark regime. Hoagland nutrient solution $(5 \mathrm{~mL})$ was applied every two weeks, and distilled water $(10 \mathrm{~mL}$ in four times) was added once a week. The cultivation lasted for 12 weeks. In the fifth week, different amounts of $\mathrm{NaCl}(0,0.18,0.54,0.9,1.35$, and $1.8 \mathrm{~g})$ were added to each cup to simulated six salinity levels $(0$,
$200,600,1000,1500$, and $2000 \mu \mathrm{S} / \mathrm{cm}$ soil conductivity). After 12 weeks of cultivation, the entire plant was dried and weighed. The relative biomass of the three species in each treatment was calculated based on the biomass of the plant cultivated individually at $0 \mu \mathrm{S} / \mathrm{cm}$ soil conductivity as a control.

\section{Statistical analysis}

One-way analysis of variance (ANOVA) was used to test the difference of electrical conductivity of rhizosphere soil of three plants species ( $R$. soongarica, $S$. glareosa, and $A$. polyrhizum), the difference of salt secretion rate of $R$. soongarica leaves under different soil salinity levels, and the difference of relative biomass of the three species. Duncan's multiple comparison was used to evaluate the differences among the treatments. Pearson's correlation analyses were conducted to examine the correlations between $R$. soongarica canopy size and the spacing between $R$. soongarica and herbs (S. glareosa or A. polyrhizum) Statistical tests were performed using SPSS version 15.0 for Windows (SPSS Inc., Chicago, IL, USA).

\section{Material statement}

This manuscript complies with institutional, national, or international guidelines and the Convention on the Trade in Endangered Species of Wild Fauna and Flora.

\section{Abbreviations}

R. soongarica: Reaumuria soongarica; S. glareosa: Stipa glareosa; A. polyrhizum: Allium polyrhizum; K. foliatum: Kalidium foliatum.

\section{Supplementary Information}

The online version contains supplementary material available at https://doi. org/10.1186/s12870-022-03457-4.

\section{Additional file 1.}

Additional file 2.

Additional file 3.

Additional file 4.

Additional file 5.

Additional file 6 .

\section{Acknowledgements}

We thank Dr. Xin Zhang and Dr. Jian Hou for English language editing. We would also like to thank Alison Beamish at the University of British Columbia for her assistance with English language and grammatical editing of the manuscript. We are grateful to the Associate Editor and two reviewers for providing valuable comments.

\section{Authors' contributions}

CW analyzed and interpreted the data. HW was a major contributor in writing the manuscript. WW, $C L, H L, L W$ gave detailed instructions to the manuscript. All authors read and approved the final manuscript. 


\section{Funding}

This work was supported by the central government guides local science and technology development fund projects (2021ZY0060) and the Science and Technology Project of Inner Mongolia (NJZZ22279). The funding body had no role in the design of the study and collection, analysis, and interpretation of data and in writing the manuscript.

\section{Availability of data and materials}

All data generated or analyzed during this study are included in this published article. Reaumuria soongarica is not a rare plant for protection. We have the permission to collect the plant samples by the local government. The voucher specimen with the collect number (Wang 20,080,705) was identified by professor Liqing Wang and deposited in Herbarium, Inner Mongolia College (HIMC).

\section{Declarations}

Ethics approval and consent to participate

Not applicable

\section{Consent for publication}

Not applicable

\section{Competing interests}

The authors declare that they have no competing interests.

\section{Author details}

${ }^{1}$ School of Ecology and Environment, Inner Mongolia University, Hohhot 010021, Inner Mongolia, China. ${ }^{2}$ Scientific Research Department, Hulunbeir College, Hulunbeir 021008, Inner Mongolia, China. ${ }^{3}$ Hulunbeir Meteorological Bureau, Hulunbeir 021000, Inner Mongolia, China.

Received: 20 April 2021 Accepted: 7 February 2022

Published online: 25 February 2022

\section{References}

1. Godsoe W, Murray R, Plank MJ. The effect of competition on species distributions depends on coexistence, rather than scale alone. Ecography. 2015;38:1071-9.

2. Richardson DM, Pyšek P. Plant invasions: merging the concepts of species invasiveness and community invasibility. Progress in Physical Geography: Earth and Environment. 2006;30:409-31.

3. Seastedt T, Pyšek P. Mechanisms of Plant Invasions of North American and European Grasslands. Annu Rev Ecol Evol Syst. 2011:42:133-53.

4. Scherrer D, Massy S, Meier S, Vittoz P, Guisan A. Assessing and predicting shifts in mountain forest composition across 25 years of climate change. Divers Distrib. 2017;23:517-28.

5. Michalet R, Maalouf JP, Choler P, Clément B, Rosebery D, Royer JM, et al. Competition, facilitation and environmental severity shape the relationship between local and regional species richness in plant communities. Ecography. 2014;38:335-45

6. Sizykh AP. Plant communities of environmental interfaces as a problem of ecology and biogeography. Biology Bulletin. 2007;34:292-6.

7. Kefu Z, Hai F, Ungar I. Survey of halophyte species in China. Plant Sci. 2002:163:491-8

8. Shan LS, Su M, Zhang ZZ, Wang Y, Wang S, Li Y. Vertical distribution pattern of mixed root systems of desert plants Reaumuria soongarica and Salsola passerina under different environmental gradients. Chinese Journal of Plant Ecology. 2018;42(4):475-86.

9. Li Z H, Chen J, ZHAO G F, Guo Y P, Kou Y X, Ma Y Z, Wang G, Ma X F. Response of a desert shrub to past geological and climatic change: A phylogeographic study of Reaumuria soongarica (Tamaricaceae) in western China. Jour of Systematics and Evol. 2012;50(4):351-61.

10. Mao W, Zhao X, Sun Z, Felton AJ, Zhang T, Li Y, et al. Limiting similarity mediates plant community niche hypervolume across a desert-steppe ecotone of Inner Mongolia. Environ Exp Bot. 2018:153:320-6.
11. Hooper DU, Dukes JS. Functional composition controls invasion success in a California serpentine grassland. J Ecol. 2010;98:764-77.

12. Naeem S, Wright JP. Disentangling biodiversity effects on ecosystem functioning: deriving solutions to a seemingly insurmountable problem. Ecol Lett. 2003;6:567-79.

13. Austrheim G, Eriksson O. Recruitment and life-history traits of sparse plant species in subalpine grasslands. Can J Bot. 2003;81:171-82.

14. Csipkés M. The examination of the profitability and competitiveness regarding the energy plantations of woody plants in the region of Észak-Alföld. Applied Studies in Agribusiness and Commerce. 2012;6:5-11.

15. Herron PM, Martine CT, Latimer AM, Leicht-Young SA. Invasive plants and their ecological strategies: prediction and explanation of woody plant invasion in New England. Divers Distrib. 2007;13:633-44.

16. Zhou HY, Bao AK, Du BQ, Wang SM. The physiological mechanisms underlying how eremophyte Reaumuria soongorica responses to severe $\mathrm{NaCl}$ stress. Pratacultural Science. 2012;29(1):71-5.

17. Xiaoning N, Minxia L, Gong Z, Sujuan X, Quandi L, Xiaoxuan J. Spatial distribution pattern and association of reaumuria soongarica and caragana roborovskyi in central loess plateau. Chin J Ecol. 2019;38(2):354-60.

18. Brockie R, Loope LL, Usher MB, Hamann O. Biological invasions of island nature reserves. Biol Cons. 1988;44:9-36.

19. Douda J, Doudová J, Hulík J, Havrdová A, Boublík K. Reduced competition enhances community temporal stability under conditions of increasing environmental stress. Ecology. 2018;99:2207-16.

20. Muthukrishnan R, Hansel-Welch N, Larkin DJ. Environmental filtering and competitive exclusion drive biodiversity-invasibility relationships in shallow lake plant communities. J Ecol. 2018;106:2058-70.

21. Botella M A, Rosado A, Bressan R A, Hasegawa P M. Plant Adaptive Responses to Salinity Stress. In: Matthew A, editor. Plant abiotic stress. Oxford: Blackwell Publishing; 2005. p. 37-70.

22. Szabados L, Kovács H, Zilberstein A, Bouchereau A. Chapter 4 - Plants in extreme environments: Importance of protective compounds in stress tolerance. Advances in Botanical Research. 2011;57:105-50.

23. Qi M, Sun T, Xue S, Yang W, Shao D, Martínez-López J. Competitive ability, stress tolerance and plant interactions along stress gradients. Ecology. 2018:99:848-57.

24. Liancourt P, Callaway RM, Michalet R. Stress tolerance and competitiveresponse abilitydetermine the outcome of biotic interactions. Ecology. 2005:86:1611-8.

25. Chaneton EJ, Mazía N, Batista WB, Rolhauser AG, Ghersa CM. Woody Plant Invasions in Pampa Grasslands: A Biogeographical and Community Assembly Perspective. In: Myster R, editor. Ecotones Between Forest and Grassland. Springer, New York: NY; 2012. p. 115-44.

26. Mazía NC, Chaneton EJ, Ghersa CM, León RJ. Limits to tree species invasion in pampean grassland and forest plant communities. Oecologia. 2001;128:594-602.

27. Vilà $M$, Weiner J. Are invasive plant species better competitors than native plant species? - evidence from pair-wise experiments. Oikos. 2004;105:229-38.

28. Funk JL, Standish RJ, Stock WD, Valladares F. Plant functional traits of dominant native and invasive species in mediterranean-climate ecosystems. Ecology. 2016;97(1):75-83.

29. Golivets M, Wallin KF. Neighbour tolerance, not suppression, provides competitive advantage to non-native plants. Ecol Lett. 2018;21:745-59.

30. Fynn R, Morris C, Kirkman K. Plant strategies and trait trade-offs influence trends in competitive ability along gradients of soil fertility and disturbance. J Ecol. 2005:93:384-94.

31. Facelli JM. Multiple Indirect Effects of Plant Litter Affect the Establishment of Woody Seedlings in Old Fields. Ecology. 1994;75:1727-35.

32. Wilson SD, Zammit CA. Tree litter and the lower limits of subalpine herbs and grasses in the Brindabella Range. ACT Austral Ecology. 1992;17:321-7.

33. Ashton IW, Lerdau MT. Tolerance to herbivory, and not resistance, may explain differential success of invasive, naturalized, and native North American temperate vines. Divers Distrib. 2007;14:169-78.

34. Diaz S, Hodgson J, Thompson K, Cabido M, Cornelissen J, Jalili A, et al. The plant traits that drive ecosystems: Evidence from three continents. J Veg Sci. 2004; 15:295-304 
35. Theoharides KA, Dukes JS. Plant invasion across space and time: factors affecting nonindigenous species success during four stages of invasion. New Phytol. 2007;176:256-73.

36. Ba L, Wang D, Hodgkinson KC, Xiao N. Competitive relationships between two contrasting but coexisting grasses. Plant Ecol. 2006;183(1):19-26.

37. Ayres P G. The aliveness of plants: the Darwins at the dawn of plant science. London: Pickering \& Chatto, 2008.

\section{Publisher's Note}

Springer Nature remains neutral with regard to jurisdictional claims in published maps and institutional affiliations.

- fast, convenient online submission

- thorough peer review by experienced researchers in your field

- rapid publication on acceptance

- support for research data, including large and complex data types

- gold Open Access which fosters wider collaboration and increased citations

- maximum visibility for your research: over 100M website views per year

At BMC, research is always in progress.

Learn more biomedcentral.com/submissions 